\title{
MICROSTRUCTURED OPTICAL FIBRE SEMICONDUCTOR METAMATERIALS
}

A. Amezcua, C.E. Finlayson, P.J.A. Sazio

Optoelectronics Research Centre (ORC), University of Southampton, Southampton

H. Fang, D. J Won, T. Scheidematel, B. Jackson, N. Baril, V. Gopalan and J. Badding

Materials Research Institute, Pennsylvania State University, University Park, PA 16802, USA

Key word to describe this work: Fibre design and fabrication, Semiconductors

Key Results: We have synthesised semiconductor wires and tubes inside microstructured optical fibres (MOFs). Their optical and electrical properties were measured.

How does the work advance the state-of-the-art? : The deposition of semiconductors inside MOF will allow the design of complex in-fibre devices.

Motivation (Problems addressed): To explore new directions in metamaterials technology.

\section{Introduction}

Microstructured optical fibres are single material optical fibres in which air-holes define the transverse structural profile. MOFs demonstrate a number of key properties when compared with conventional silica fibres, including broad band single mode guidance, nonlinear properties, and photonic band gap effects [1]. The inclusion of materials such as semiconductors, metals or polymers within the microscale or nanoscale holes of MOFs presents radically novel electronic, photonic and plasmonic degrees of freedom for the exploration of new directions in metamaterials technology. This allows the design of complex optical fibre devices with exceptional tuneable properties. Furthermore, the inclusion of semiconductors to nanoscale MOFs holes will bring together fibre optics technology with the growing field of ultra small semiconductor nanowires [2] for the generation and manipulation of light.

\section{Results}

Our initial device structures were fabricated by filling a $2 \mu \mathrm{m}$ silica capillary with silicon using a high pressure chemical deposition technique. As can be seen in the SEM picture of the resulting optical fibre (Figure 1), silicon was deposited within the capillary void forming a uniform ring with a wall thickness of $600 \mathrm{~nm}$.

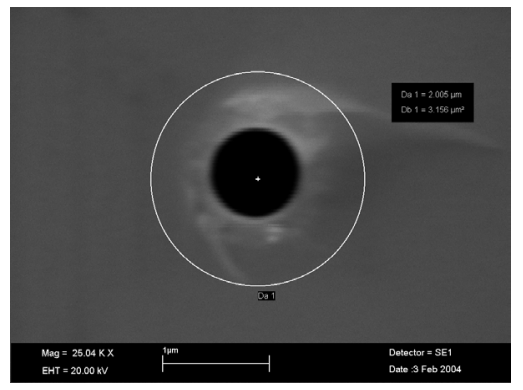

Fig. 1. SEM micrograph of a silica capillary fibre that has been filled with silicon. The circle indicates the original hole size ( 2 microns).

To explore its optical properties, ASE radiation $(1530-1570 \mathrm{~nm})$ was launched into the silicon optical fibre which was immersed in index matching fluid to remove any cladding light, and the resulting near field output was imaged onto an infrared camera. As it can be seen in Figure 2, the experiments reveal strong evidence that a significant fraction of ASE has been guided by the silicon core.

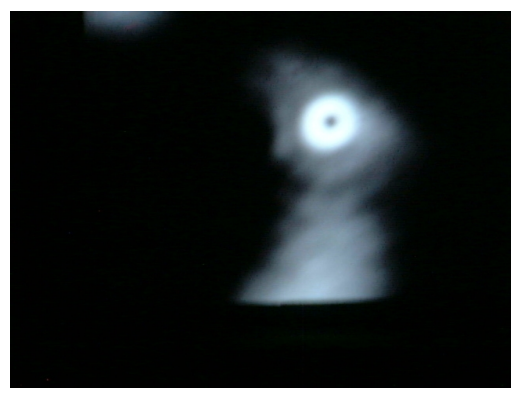

Fig. 2. Near field output of the ASE radiation guided by the silicon filled optical fibre 
In order to gain further insight into the guiding properties of semiconductor optical fibres, beam propagation method (BMP) software was used to determine the mode profile and the number of optical modes allowed to propagate within the silicon core at $1.55 \mu \mathrm{m}$. As expected from the high refractive index contrast between silicon and silica, the simulations reveal the multimode behaviour of the optical fibre at $1.55 \mu \mathrm{m}$ and the strong energy confinement in the semiconductor core (Figure 3).

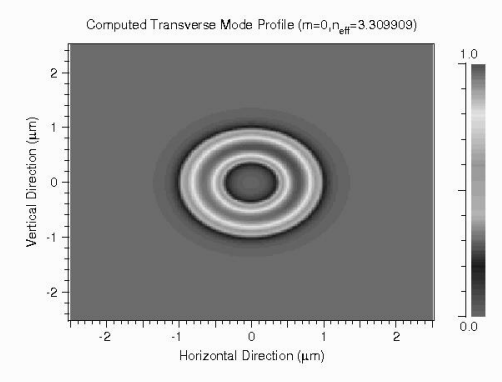

Fig. 3. Computed fundamental mode distribution. The scale bar indicates the power density.

Further device characterisation was undertaken by investigating the electron transport properties of silicon filled capillaries. For these experiments, silicon was deposited inside $5 \mu \mathrm{m}$ internal diameter silica capillaries, and the filled fibres prepared to allow the silicon core to emerge from both ends of the silica capillary. A 200 $\mathrm{nm}$ thick Aluminium layer was then thermally evaporated onto the ends of the fibre, followed by an annealing process to activate the ohmic contacts.

An electrical prober station was used to investigate the current-voltage characteristics of two samples. Ohmic behaviour was observed in both cases with the resistances (as measured from the gradient) being $5 \times 10^{10} \Omega$ and $3.75 \times 10^{10} \Omega$, which are consistent with the respective sample lengths of $24 \mathrm{~mm}$ and $18 \mathrm{~mm}$. The sample resistivity was calculated to have a value of $0.4 \Omega \mathrm{cm}$, thus corresponding to a dopant concentration of $\sim 10^{16} \mathrm{~cm}^{-3}[4]$.

Figure 4 shows the result of 3-terminal electrical measurements on one of the fibres, with the gate voltage on a central gate contact of $+/-100 \mathrm{~V}$. We see a clear increase in the I-V gradient, which implies a decrease in resistance as the voltage increases. Such behaviour is expected for material in which the electrons are the majority carrier.

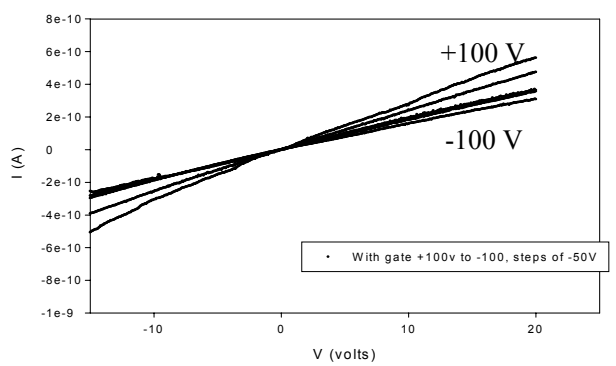

Fig. 4. Transport properties of a silicon filled optical fibre. The source-drain characteristics are shown as a function of gate voltage $(+100$ to $-100 \mathrm{~V}$ in $50 \mathrm{~V}$ steps).

\section{Future directions}

The deposition of semiconductors within microstructured optical fibres will open new possibilities for next generation photonic, electronic and plasmonic based device structures and sensors. For example, it opens the possibility of considering in-fibre semiconductor lasers [4], modulators, switches, and high-speed tuneable photonic bandgap devices. Furthermore, if the fibre incorporates nanoscale holes, the impregnation of semiconductors will allow the exploration of semiconductor quantum confinement properties. Finally, due to the wide transparency window of semiconductors at infrared wavelengths, our new type of optical fibres present a large range of opportunities for mid-infrared delivery systems and sources.

\section{References}

[1] P. Russell, Science 299, 358-362 (2003).

[2] A. M. Morales and C. M. Lieber, Science 279, 208-211 (1998).

[3] http://www.ee.byu.edu/cleanroom/ResistivityCal.phtml [4] Kornreich et al., J.Light.Tech.14, 1674-1676 (1996). 